Research paper

\title{
Bacterial cellulose membranes as drug delivery systems: An in vivo skin compatibility study
}

\author{
I.F. Almeida ${ }^{\text {a,* }}$, T. Pereira ${ }^{b}$, N.H.C.S. Silva ${ }^{c}$, F.P. Gomes ${ }^{c}$, A.J.D. Silvestre ${ }^{c}$, C.S.R. Freire ${ }^{c}$, J.M. Sousa Lobo ${ }^{a}$, \\ P.C. Costa ${ }^{a}$ \\ a Department of Drug Sciences, University of Porto, Portugal \\ ${ }^{\mathrm{b}}$ Centro de Dermatologia Epidermis, Instituto CUF, Porto, Portugal \\ ${ }^{\mathrm{c}}$ CICECO and Department of Chemistry, University of Aveiro, Portugal
}

\section{A R T I C L E I N F O}

Article history:

Received 22 May 2013

Accepted in revised form 8 August 2013

Available online $\mathrm{xxxx}$

\section{Keywords:}

Bacterial cellulose

Skin hydration

Skin compatibility

Gluconoacetobacter sacchari

Drug delivery

Skin irritation

\begin{abstract}
A B S T R A C T
Bacterial cellulose (BC) is a highly pure form of cellulose, produced in the form of a swollen membrane by several bacteria that demonstrated to be able to modulate the skin release of model drugs. In the present study, the skin irritation potential of BC was evaluated in human subjects. BC membranes with and without glycerin (acting as plasticizer) were tested. No significant differences were observed for transepidermal water loss (TEWL) measurements in comparison with negative control, 2 and $24 \mathrm{~h}$ after patch removal, which is an indicator of an absence of barrier disruption. Similar results were found for erythema. Clinical scores were zero at both times for all volunteers, with the exception of five volunteers that exhibited weak reactions. BC with glycerin provided a skin moisturizing effect statistically higher than the negative control $(p=0.044)$, which was not observed for $\mathrm{BC}$ alone. The good skin tolerance found after a single application under occlusion reinforces the putative interest of $\mathrm{BC}$ membranes as supports for drug topical delivery. Besides modifying the mechanical properties, the inclusion of glycerin results in a skin moisturizing effect which could be clinically relevant for the treatment for skin diseases characterized by dryness, such as psoriasis and atopic dermatitis.
\end{abstract}

(c) 2013 Elsevier B.V. All rights reserved.

\section{Introduction}

Bacterial cellulose (BC), also known as biocellulose, is an extracellular polysaccharide produced by several bacteria of the genera Gluconacetobacter, Agrobacter, Sarcina, among others, in the form of a wet membrane ( $\sim 99 \%$ water) in the interface air/culture medium [1]. Bacterial cellulose presents high purity and water absorption capacity, as well as unique mechanical properties, good permeability, and resistance to degradation [2-4]. Most of these properties arise from $B C$ peculiar tridimensional nanofibrillar network. Due to its singular properties and biocompatibility [2,5], BC has triggered considerable interest in several fields, but particularly in the biomedical area, as for example, in wound dressing for skin burns [2,5] and in artificial blood vessels for microsurgery [6]. Moreover, the peculiar nanofibrillar structure of BC should represent a suitable macromolecular support for inclusion of drugs and therefore for the development of specific controlled release systems. Previous studies documented the ability of BC

\footnotetext{
* Corresponding author. Laboratory of Pharmaceutical Technology, Department of Drug Sciences, Faculty of Pharmacy, University of Porto, Rua Jorge Viterbo Ferreira no. 228, 4050-313 Porto, Portugal. Tel.: +351 220428621.

E-mail address: ifalmeida@ff.up.pt (I.F. Almeida).
}

membranes to modulate the release and bioavailability of model drugs for percutaneous administration [7,8], and hence, they were proposed as supports for topical or transdermal drug delivery. Therapeutic feasibility is dependent on the skin compatibility of these supports; however, little information is available concerning the skin compatibility and irritation potential of BC-based biomaterials. Cytotoxicity studies have been conducted with human cells [9-11]. However, reports regarding in vivo biocompatibility are rather scarce and mainly used mice surgeries [9,11,12]. Clinical tests have been conducted with commercial biocellulose films mainly for wound healing effect [13] which account for their dermal compatibility, but this parameter has not been objectively characterized. Only one study described the absence of skin irritation when a biocellulose mask was applied for $24 \mathrm{~h}$ to the arm of human volunteers [14]. To our knowledge, the present study is the first report of human skin irritation evaluation of $\mathrm{BC}$ membranes under occlusion. Patch testing after single application is a widely used procedure to evaluate acute irritant reactions [15]. In this methodology, occlusion is achieved by means of an aluminum chamber in order to exaggerate exposition conditions. A $24 \mathrm{~h}$ patch test with a follow-up reading at $24 \mathrm{~h}$ after patch removal is recommended as optimal procedure [16]. The evaluation of irritant reactions can be accomplished with objective and 
subjective methods. Visual scoring, although subjective, can be a sensitive, reliable, and reproducible method [17]. Several bioengineering techniques have been developed to provide objective and quantitative data. Transepidermal water loss (TEWL) is considered to be the first choice to evaluate slight skin reactions as it detects disrupted epidermal barrier that results in a higher loss of TEWL [18]. However, water barrier function and water evaporation from the skin are neither felt nor seen, and therefore, findings based on TEWL measurements are indirect and not automatically clinically relevant [19]. In this work, the evaluation of irritant reactions was carried out with a combined approach in order to cover the different features of skin irritation. The aim of the present study was to evaluate the skin irritant potential of $\mathrm{BC}$ membranes (with and without glycerin) and characterize their skin moisturizing effect.

\section{Materials and reagents}

Glucose (96\% purity), glycerin (99.5\%), and $\mathrm{Na}_{2} \mathrm{HPO}_{4}$ were purchased from Sigma-Aldrich. Yeast extract and bacteriological peptone were purchased from Himedia, and citric acid $(99.6 \%$ purity) was obtained from Acros Organics. Glycerin solution (99\%) was purchased from Fagron, and sodium lauryl sulfate (SLS, 99\% purity) was obtained from Fluka. All other chemicals were of analytical grade. Patch test was performed with aluminum chambers (12 mm, Finn Chambers ${ }^{\circledR}$, Epitest) fixed with adhesive tape (Scanpore ${ }^{\circledR}$, Norgeplaster).

\section{1. $B C$ production and purification}

BC membranes were produced using Gluconacetobacter sacchari [3]. The pre-inocula were prepared at $30^{\circ} \mathrm{C}$ during $48 \mathrm{~h}$, in static conditions, in Hestrin and Shramm (HS) liquid medium (20 g/L glucose, $5 \mathrm{~g} / \mathrm{L}$ peptone, $5 \mathrm{~g} / \mathrm{L}$ yeast extract, $2.7 \mathrm{~g} / \mathrm{L} \mathrm{Na}_{2} \mathrm{HPO}_{4}, 1.15 \mathrm{~g} / \mathrm{L}$ citric acid, agar, $15 \mathrm{~g} / \mathrm{L}, \mathrm{pH} 5)$, before inoculation $(10 \% \mathrm{v} / \mathrm{v})$ of $50 \mathrm{~mL}$ into $500 \mathrm{~mL}$ liquid production medium in Erlenmeyer flasks. The flasks were kept at $30^{\circ} \mathrm{C}$, in static conditions, for $96 \mathrm{~h}$. After the incubation period, the $\mathrm{BC}$ membranes were withdrawn from the culture medium and treated with $0.5 \mathrm{M} \mathrm{NaOH}$ at $90^{\circ} \mathrm{C}$ for $30 \mathrm{~min}$. This procedure was repeated three times in order to eliminate attached cells [20]. Then, the membranes were washed with distilled water to remove components of the culture medium and other residues until its whitening and reaching $\mathrm{pH}$ 7.0.

\subsection{Preparation of $B C$ and $B C$-glycerin (BC-Gly) membranes}

BC discs ( $7 \mathrm{~cm}$ diameter) were prepared from $8 \mathrm{~mm}$ thick wet membranes ( $\sim 167 \mathrm{mg}$ dry weight). BC discs for testing were placed in a Petri dish and dried at $40^{\circ} \mathrm{C}$ in a ventilated oven for $16 \mathrm{~h}$; BC membranes were previously drained down to $40 \%$ water content by hand-pressing between two acrylic plates at room temperature. BC-Gly membranes were prepared by soaking BC discs in $8 \mathrm{~mL}$ of an aqueous buffered solution ( $\mathrm{pH} 7.4$ ) of $1 \%$ glycerin, during $48 \mathrm{~h}$ at room temperature to assure complete absorption of glycerin. After the total absorption of the solution, BC-Gly membranes were placed over a Petri dish and dried as described above. The dried BC (about 5\% residual water) and BC-Gly membranes (about 5\% residual water and $32 \%$ glycerin) were kept in a desiccator until their use.

\subsection{Characterization of $B C$ and $B C$-glycerin (BC-Gly) membranes morphology and mechanical performance}

Scanning Electron Microscopy (SEM) of the surface and crosssection of dried BC and BC-Gly membranes were performed using a Hitachi SU-70 instrument operating at $4 \mathrm{kV}$. BC and BC-Gly membranes were placed in an appropriated steel support and covered with evaporated carbon. For cross-section analysis, the samples were previously broken with liquid nitrogen to expose appropriated cross-section regions.

Tensile assays were performed on an Instron machine 5966 Series, using a load cell of $500 \mathrm{~N}$, operating at a deformation rate of $10 \mathrm{~mm} / \mathrm{min}$, under ambient conditions. At least 5 specimens were tested for each composite. Tensile strength, tensile modulus, and elongation at break were calculated using the Bluehill 3 Material Testing software.

\subsection{In vivo evaluation}

Fifteen healthy individuals ( 12 female and 3 male) with a mean age of $31.1 \pm 9.9$ years participated in this study, and written informed consent was obtained from all volunteers. BC and BC-Gly samples were tested. SLS $(2 \% \mathrm{w} / \mathrm{v})$ was the positive control, and an empty chamber was used as negative control. An aqueous glycerin solution was also tested. Test sites were randomized between volunteers. The products were applied in the inner forearm (Fig. 1), and the patches were removed after $24 \mathrm{~h}$. The visual assessment of the degree of irritation was made at 2 and $24 \mathrm{~h}$ after patch removal and graded by an experienced dermatologist according to a 5-level reference scale [21]. In the same time intervals, TEWL measurements were performed with a Tewameter $^{\circledR}$ (TM 210, Courage + Khazaka, Germany), and erythema was evaluated with a Colorimeter (CR-400, Minolta, Japan). Skin moisturizing effect was assessed with a Corneometer ${ }^{\circledR}$ (CM 825, MPA $9^{\circledR}$, Courage-Khazaka, Germany). All measurements were made in a draught-free room, with controlled temperature $\left(18.6-22.5^{\circ} \mathrm{C}\right)$ and relative humidity (41.4-60\%). The volunteers were asked not to apply any topical products in the forearms $24 \mathrm{~h}$ before the beginning and throughout the test period. Additionally, solar exposure and use of occlusive clothes on the test area were forbidden. The study was carried out in accordance with The Code of Ethics of the World Medical Association (Declaration of Helsinki) for experiments involving humans and was approved by the ethics committee of the Faculty of Pharmacy of the University of Porto.

\subsection{Statistical analysis}

Statistical evaluation of erythema, moisturizing effect, and TEWL data (variation from basal values) was performed using ANOVA. Post hoc comparisons with negative control were performed with Dunnett test. Statistical analysis was conducted with IBM SPSS Statistics $21(\alpha=0.05)$.

\section{Results and discussion}

\subsection{Membrane characterization}

BC and BC-Gly membranes were considered in this study, since glycerin is normally used as a plasticizer, to increase malleability, as well as the swelling (by promoting water holding and retention) of topical delivery systems [7,8]. Fig. 2 displays the visual aspect of BC and BC-Gly dried membranes. BC-Gly membranes are slightly more translucent than pure $\mathrm{BC}$ membranes and also quite homogeneous which clearly indicates a good dispersion of glycerin inside the BC nano- and microfibrills network surely due to the establishment of strong interactions between the two $\mathrm{OH}$ rich structures.

The surface and cross-section morphology of dried BC and BC-Gly membranes was assessed by SEM (Fig. 3). The surface micrographs of both $\mathrm{BC}$ and $\mathrm{BC}-\mathrm{Gly}$ membranes showed the characteristic tridimensional nanofibrillar network of $\mathrm{BC}$ matrices; however, in BC-Gly, it is less compacted, certainly because of the presence of glycerin that limited the extent of collapse of the 

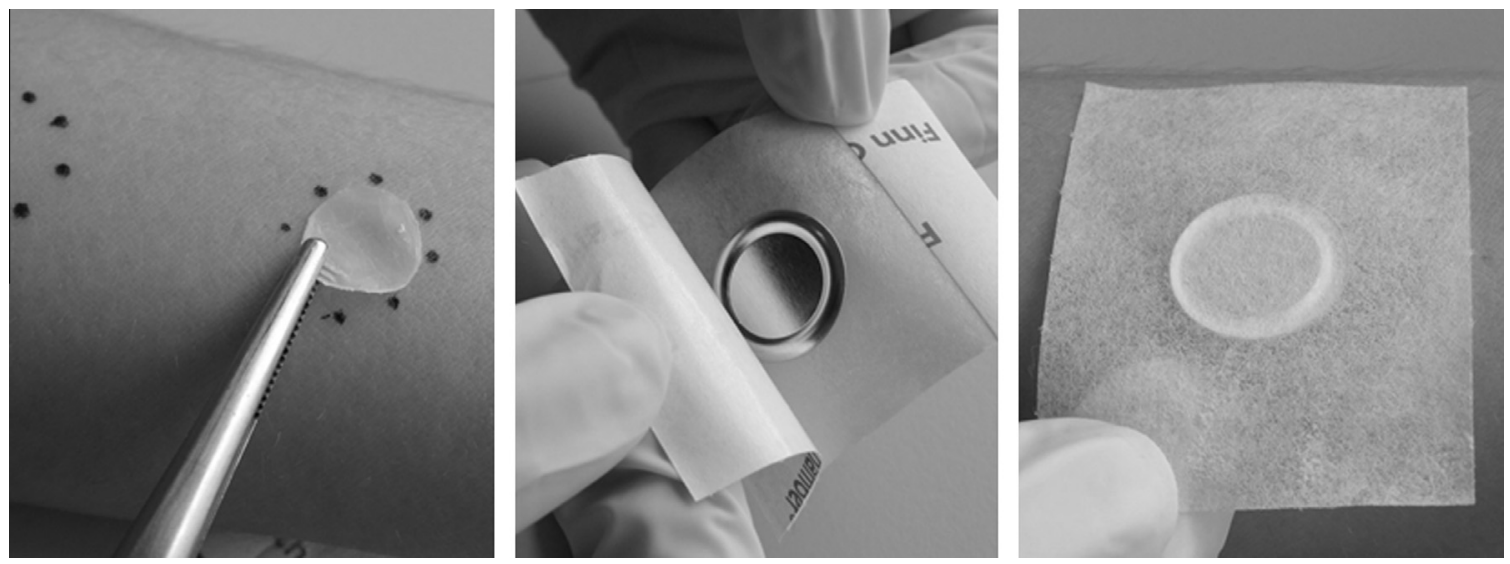

Fig. 1. Sequential steps of the patch test using Finn Chambers ${ }^{\circledR}$.

tridimensional structure of $\mathrm{BC}$ during drying. Glycerin is retained between the cellulose nanofibers, reducing the intermolecular attraction forces and therefore increasing the distance between them. This behavior is particularly noticeable on the cross-section images where the typical lamellar morphology is much more expanded for BC-Gly rather than for BC.

In terms of mechanical properties, as intended, the addition of glycerin to the BC membranes increased considerably their malleability as observed by the increment in the elongation at break and by the corresponding decrease in the Young modulus of BC-Gly when compared to BC (Table 1). This feature is quite significant in terms of the clinical application of BC membranes since bendable type materials are easier to fix and to use.

\subsection{In vivo tests}

Significant differences were observed for TEWL and erythema for the positive control (SLS), and the irritation scores were different from zero for all volunteers, for both measuring times. These findings confirm the validity of the procedure undertaken. Regarding BC membranes, no significant differences were observed for TEWL measurements in comparison with negative control, 2 and $24 \mathrm{~h}$ after patch removal (Fig. 4), which is an indicator of an absence of barrier disruption. Similar results were found for erythema (Fig. 5). Clinical scores were zero at both times for all volunteers, with the exception of five volunteers that exhibited weak reactions (scores 1 or 2 ). From these, two volunteers also presented weak reactions to the negative control (Table 2). None of the individuals reported subjective irritation symptoms like stinging or itching. Taken together, these results confirm the good skin tolerance of both BC and BC-Gly membranes.

BC-Gly membranes provided a skin moisturizing effect, statistically higher than the negative control $(p=0.044) 2 \mathrm{~h}$ after patch removal which was not observed for BC alone (Fig. 6). Occlusion

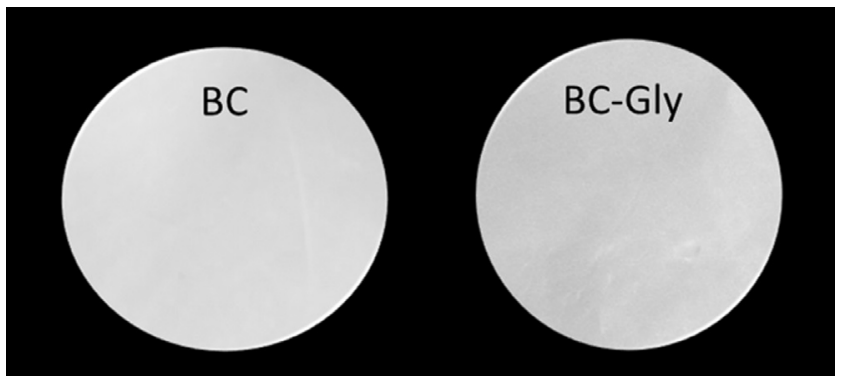

Fig. 2. Visual aspect of dried BC and BC-Gly membranes. provided by aluminum chambers may have potentiated this effect; however, the moisturizing ability observed was similar to that exhibited by an aqueous solution of glycerin, a well-known moisturizer widely used in skin care preparations. Besides, occlusion of the untreated site did not induce a relevant increase in skin hydration (Fig. 6). Skin hydration decreased $24 \mathrm{~h}$ after patch removal. The persistence of the moisturizing effect could be evaluated after long-term application. The role of glycerin in skin moisturization is well documented [22] and has been traditionally attributed to its highly hygroscopic nature associated with the presence of three hydroxyl groups. Glycerin present in BC-Gly membranes diffused into the stratum corneum and retained water in this skin layer. Among other biological actions, anti-irritant properties and recovery of the skin barrier functions have also been reported for this polyol [23]. For these reasons, formulations containing this moisturizer have been tested in the treatment for dermatosis with damaged barrier such as atopic dermatitis and in the treatment of dry skin conditions such as winter and senile xerosis [24-26]. As a consequence, it may be reasonably postulated the application of BG-Gly membranes in the therapy of skin lesions where moisturization ameliorates the clinical symptoms. This effect can also be complement with the therapeutic action of a drug loaded on BC membrane such as an anti-inflammatory or an antipruritic compound. Besides therapeutic application, BC membranes have been studied for cosmetic use [14]. It was reported that a facial BC mask applied for 5 min promoted an increase in skin moisture. This effect was attributed to the water content of the mask that augmented the water uptake by the skin. This mechanism cannot explain the effect observed in this study since the BC membranes used were previously dried and their water content was low $(\leqslant 10 \%)$. In the above mentioned study, the majority of the volunteers rated the cellulose mask comfortable to use [14]. The mask also adhered well to the skin. Consumer/patient compliance is an important issue regarding clinical efficacy. It has been reported that failure to comply with prescribed topical treatment can be as high as $60 \%$, especially in chronic dermatosis such as psoriasis [27]. Poor cosmetic acceptability has been recognized as one of the reasons for non-adherence [27]. The results obtained in the study with a cellulose mask [14] cannot be fully transposed to the system studied in this work, since different preparation methods were used. However, it provides good indicators regarding cosmetic acceptability of $\mathrm{BC}$-based preparations.

The ability of $\mathrm{BC}$ membranes to modulate the release of drugs can reduce the number of applications which offers a more simple and convenient therapeutic regimen especially considering chronic dermatosis. Membrane flexibility given by glycerin is highly valuable considering the need for adaptation to skin irregular 

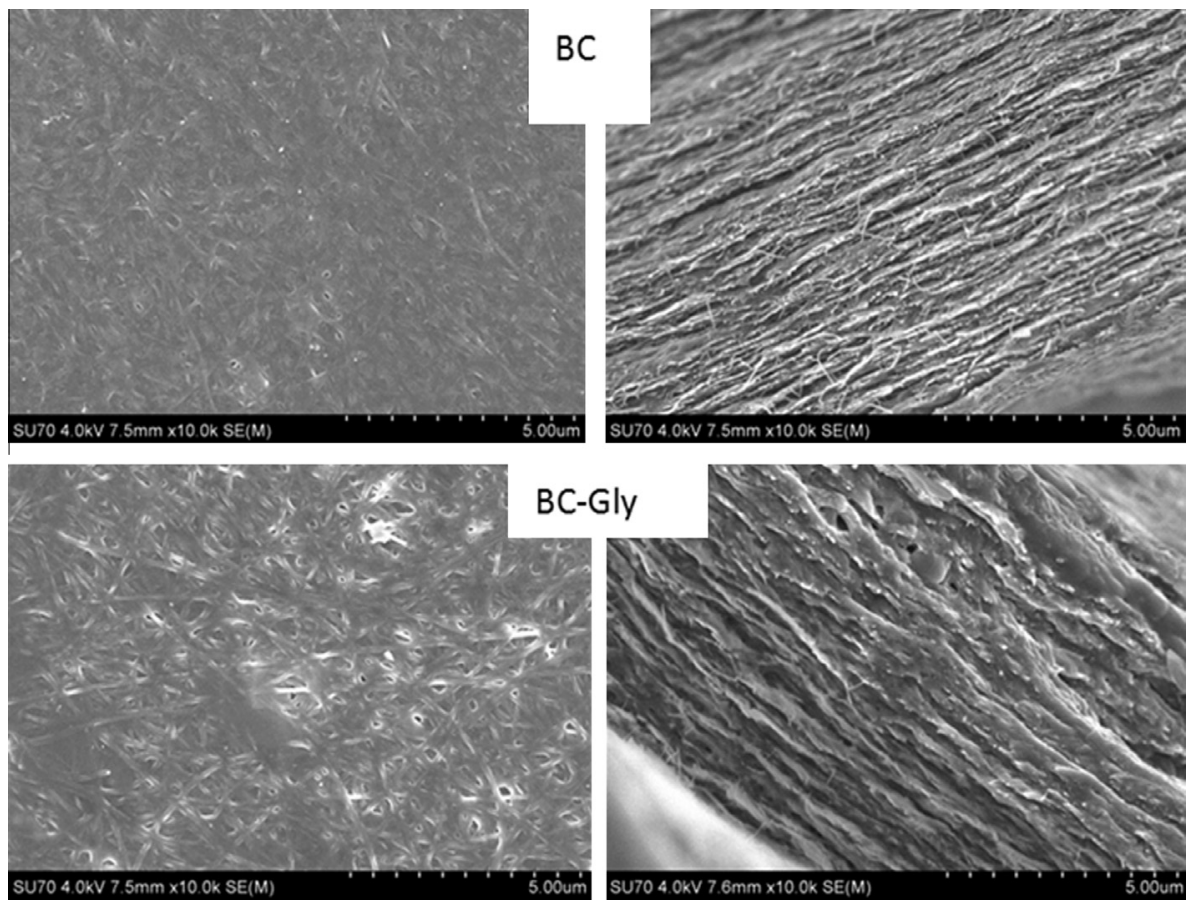

Fig. 3. Surface (left) and cross-section (right) SEM images of oven dried BC and BC-Gly membranes.

Table 1

Young modulus, tensile strength and elongation at break of BC and BC-Gly membranes, obtained from mechanical tensile assays.

\begin{tabular}{llll}
\hline Sample & $\begin{array}{l}\text { Young modulus } \\
(\mathrm{MPa})\end{array}$ & $\begin{array}{l}\text { Elongation at } \\
\text { break }(\%)\end{array}$ & $\begin{array}{l}\text { Tensile strength } \\
(\mathrm{MPa})\end{array}$ \\
\hline BC & $19132.32 \pm 1286.15$ & $2.26 \pm 0.73$ & $271.93 \pm 62.06$ \\
BC-Gly & $9412.06 \pm 747.70$ & $6.17 \pm 0.73$ & $360.30 \pm 51.00$ \\
\hline
\end{tabular}

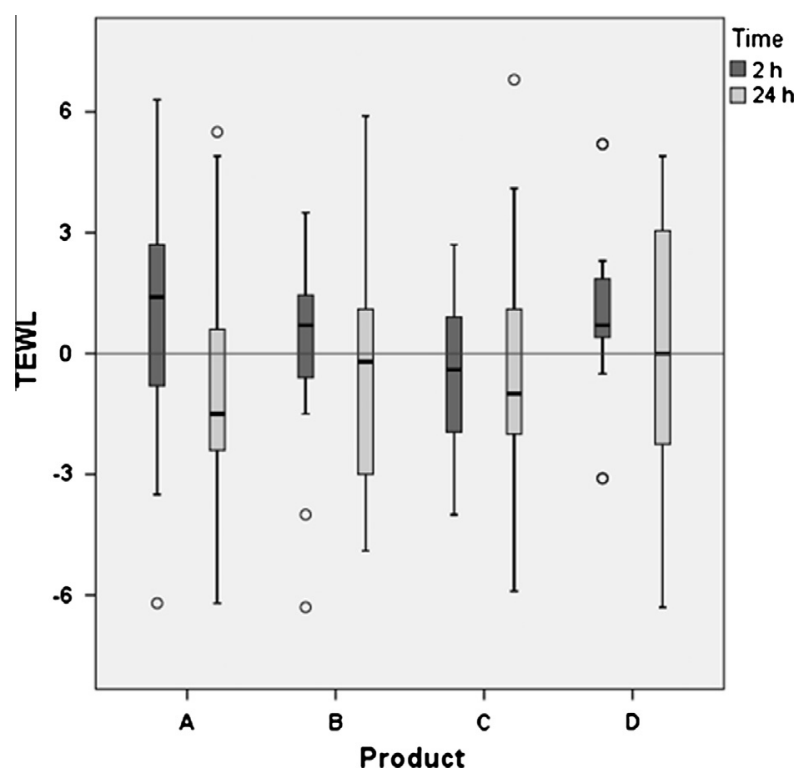

Fig. 4. TEWL measurements $2 \mathrm{~h}$ and $24 \mathrm{~h}$ after patch removal. (A) BC membrane; (B) BC-Gly membrane; (C) Glycerin solution, and (D) Negative control. Outliers are represented as circles.

areas. The membranes can also be cut (or is situ conformable) into the desired shape and dimensions to fit the target area.

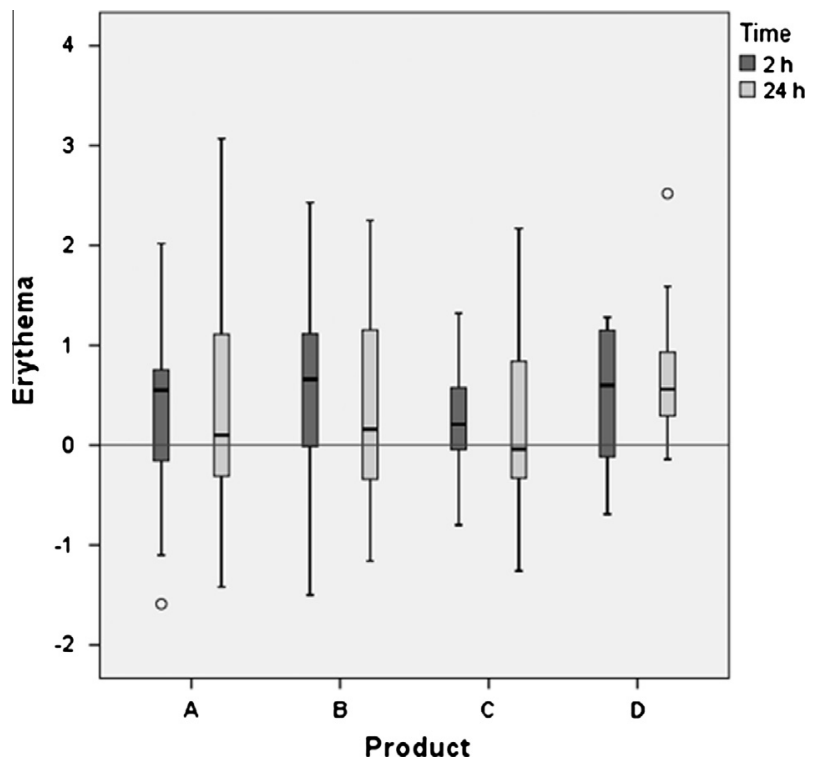

Fig. 5. Erythema measurements $2 \mathrm{~h}$ and $24 \mathrm{~h}$ after patch removal. (A) BC membrane; (B) BC-Gly membrane; (C) Glycerin solution, and (D) Negative control Outliers are represented as circles.

Another critical issue regarding the use of topical semisolid formulations is the loss of material due to contact with garments or surfaces [8]. Regarding dose accuracy, for semisolids, the amount of drug used and application area are patient-dependent and therefore are not accurate and vary widely with the patient perception. In a topical delivery system based on $\mathrm{BC}$, the dose can be precisely defined by the membrane area applied to the skin, and the characteristics of such a system prevent any loss of drug after application. Advantages over traditional transdermal patches can also be pointed out, such as a lower number of layers which render lower production costs. 
Table 2

Irritation scores obtained in the patch test.

\begin{tabular}{|c|c|c|c|c|c|c|c|c|c|c|}
\hline \multirow[t]{2}{*}{ Score/Time } & \multicolumn{2}{|c|}{ Untreated } & \multicolumn{2}{|l|}{$\mathrm{BC}$} & \multicolumn{2}{|c|}{ BC-Gly } & \multicolumn{2}{|l|}{ SLS } & \multicolumn{2}{|c|}{ Glycerin solution } \\
\hline & $2 \mathrm{~h}$ & $24 \mathrm{~h}$ & $2 \mathrm{~h}$ & $24 \mathrm{~h}$ & $2 \mathrm{~h}$ & $24 \mathrm{~h}$ & $2 \mathrm{~h}$ & $24 \mathrm{~h}$ & $2 \mathrm{~h}$ & $24 \mathrm{~h}$ \\
\hline 0 & 13 & 15 & 11 & 14 & 11 & 14 & 0 & 0 & 14 & 15 \\
\hline 1 & 2 & 0 & 3 & 1 & 2 & 1 & 1 & 2 & 1 & 0 \\
\hline 2 & 0 & 0 & 1 & 0 & 2 & 0 & 4 & 2 & 0 & 0 \\
\hline 3 & 0 & 0 & 0 & 0 & 0 & 0 & 0 & 2 & 0 & 0 \\
\hline 4 & 0 & 0 & 0 & 0 & 0 & 0 & 3 & 6 & 0 & 0 \\
\hline 5 & 0 & 0 & 0 & 0 & 0 & 0 & 7 & 3 & 0 & 0 \\
\hline
\end{tabular}

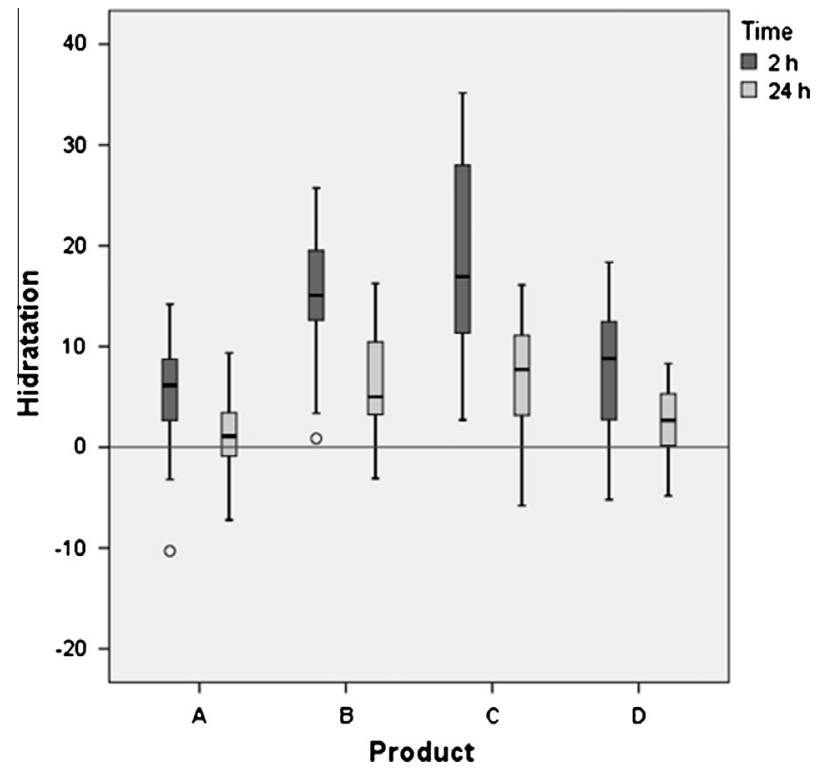

Fig. 6. Capacitance measurements $2 \mathrm{~h}$ and $24 \mathrm{~h}$ after patch removal. (A) BC membrane; (B) BC-Gly membrane; (C) Glycerin solution, and (D) Negative control. Outliers are represented as circles.

\section{Conclusions}

The good skin tolerance found after $24 \mathrm{~h}$ patch test reinforces the putative interest of $\mathrm{BC}$ membranes as supports for topical or transdermal drug delivery. It should be mentioned, however, that use tests are needed to confirm skin compatibility under normal use conditions. The inclusion of glycerin besides increasing malleability results in a skin moisturizing effect, which could be clinically relevant for the treatment for skin diseases characterized by dryness such as psoriasis and atopic dermatitis.

\section{Acknowledgement}

The authors are grateful to CICECO (Pest-C/CTM/LA0011/2013).

\section{References}

[1] D. Klemm, B. Heublein, H.P. Fink, A. Bohn, Cellulose: fascinating biopolyme and sustainable raw material, Angew. Chem. Int. Ed. 44 (2005) 3358-3393.

[2] W. Czaja, A. Krystynowicz, S. Bielecki, R.M. Brown Jr., Microbial cellulose - the natural power to heal wounds, Biomaterials 27 (2006) 145-151.

[3] M. Iguchi, S. Yamanaka, A. Budhiono, Bacterial cellulose - a masterpiece of nature's arts, J. Mater. Sci. 35 (2000) 261-270.

[4] E. Trovatti, L.S. Serafim, C.S.R. Freire, A.J.D. Silvestre, C.P. Neto, Gluconacetobacter sacchari: an efficient bacterial cellulose cell-factory, Carbohydr. Polym. 86 (2011) 1417-1420.

[5] W.K. Czaja, D.J. Young, M. Kawecki, R.M. Brown Jr., The future prospects of microbial cellulose in biomedical applications, Biomacromolecules 8 (2007) 1-12.

[6] D. Klemm, D. Schumann, U. Udhardt, S. Marsch, Bacterial synthesized cellulose - artificial blood vessels for microsurgery, Prog. Polym. Sci. 26 (2001) 15611603.
[7] E. Trovatti, C.S. Freire, P.C. Pinto, I.F. Almeida, P. Costa, A.J. Silvestre, C.P. Neto, C. Rosado, Bacterial cellulose membranes applied in topical and transdermal delivery of lidocaine hydrochloride and ibuprofen: in vitro diffusion studies, Int. J. Pharm. 435 (2012) 83-87.

[8] E. Trovatti, N.H. Silva, I.F. Duarte, C.F. Rosado, I.F. Almeida, P. Costa, C.S. Freire, A.J. Silvestre, C.P. Neto, Biocellulose membranes as supports for dermal release of lidocaine, Biomacromolecules 12 (2011) 4162-4168.

[9] L.N. Fu, Y. Zhang, C. Li, Z.H. Wu, Q. Zhuo, X. Huang, G.X. Qiu, P. Zhou, G. Yang, Skin tissue repair materials from bacterial cellulose by a multilayer fermentation method, J. Mater. Chem. 22 (2012) 12349-12357.

[10] S.I. Jeong, S.E. Lee, H. Yang, Y.H. Jin, C.S. Park, Y.S. Park, Toxicologic evaluation of bacterial synthesized cellulose in endothelial cells and animals, Mol. Cell Toxicol. 6 (2010) 373-380.

[11] P.N. Mendes, S.C. Rahal, O.C.M. Pereira-Junior, V.E. Fabris, S.L.R. Lenharo, J.F. de Lima-Neto, F.D. Landim-Alvarenga, In vivo and in vitro evaluation of an Acetobacter xylinum synthesized microbial cellulose membrane intended for guided tissue repair, Acta Vet. Scand. 51 (2009).

[12] L.S. Scuro, P.U. Simioni, D.L. Grabriel, E.E. Saviani, L.V. Modolo, W.M. Tamashiro, I. Salgado, Suppression of nitric oxide production in mouse macrophages by soybean flavonoids accumulated in response to nitroprusside and fungal elicitation, BMC Biochem. 5 (2004) 5.

[13] E. Lenselink, A. Andriessen, A cohort study on the efficacy of a polyhexanidecontaining biocellulose dressing in the treatment of biofilms in wounds, J. Wound Care 20 (2011). 534, 536-539.

[14] T. Amnuaikit, T. Chusuit, P. Raknam, P. Boonme, Effects of a cellulose mask synthesized by a bacterium on facial skin characteristics and user satisfaction, Med. Dev. 4 (2011) 77-81.

[15] S.A. Flannigan, R.E. Smith, J.P. McGovern, Intraregional variation between contact irritant patch test sites, Contact Dermatitis 10 (1984) 123-124.

[16] H. Kanto, K. Washizaki, M. Ito, K. Matsunaga, H. Akamatsu, K. Kawai, N. Katoh, M. Natsuaki, I. Yoshimura, H. Kojima, Y. Okamoto, M. Okuda, H. Kuwahara, M. Sugiyama, S. Kinoshita, F. Mori, Optimal patch application time in the evaluation of skin irritation, J. Dermatol. (2013).

[17] D. Basketter, F. Reynolds, M. Rowson, C. Talbot, E. Whittle, Visual assessment of human skin irritation: a sensitive and reproducible tool, Contact Dermatitis 37 (1997) 218-220.

[18] J. Aramaki, I. Effendy, R. Happle, S. Kawana, C. Loffler, H. Loffler, Which bioengineering assay is appropriate for irritant patch testing with sodium lauryl sulfate? Contact Dermatitis 45 (2001) 286-290.

[19] R.A. Tupker, C. Willis, E. Berardesca, C.H. Lee, M. Fartasch, T. Agner, J. Serup, Guidelines on sodium lauryl sulfate (SLS) exposure tests. A report from the standardization group of the European society of contact dermatitis, Contact Dermatitis 37 (1997) 53-69.

[20] S. Bae, Y. Sugano, M. Shoda, Improvement of bacterial cellulose production by addition of agar in a jar fermentor, J. Biosci. Bioeng. 97 (2004) 33-38.

[21] N. Jibry, S. Murdan, In vivo investigation, in mice and in man, into the irritation potential of novel amphiphilogels being studied as transdermal drug carriers, Eur. J. Pharm. Biopharm. 58 (2004) 107-119.

[22] M. Lodén, W. Wessman, The influence of a cream containing 20\% glycerin and its vehicle on skin barrier properties, Int. J. Cosmet. Sci. 23 (2001) 115-119.

[23] J.W. Fluhr, M. Gloor, L. Lehmann, S. Lazzerini, F. Distante, E. Berardesca, Glycerol accelerates recovery of barrier function in vivo, Acta Derm. Venereol. 79 (1999) 418-421.

[24] M. Loden, A.C. Andersson, C. Andersson, T. Frodin, H. Oman, M. Lindberg, Instrumental and dermatologist evaluation of the effect of glycerine and urea on dry skin in atopic dermatitis, Skin Res. Technol.: Off. J. Int. Soc. Bioeng. Skin 7 (2001) 209-213.

[25] M. Loden, A.C. Andersson, C. Anderson, I.M. Bergbrant, T. Frodin, H. Ohman, M.H. Sandstrom, T. Sarnhult, E. Voog, B. Stenberg, E. Pawlik, A. PreislerHaggqvist, A. Svensson, M. Lindberg, A double-blind study comparing the effect of glycerin and urea on dry, eczematous skin in atopic patients, Acta Derm. Venereol. 82 (2002) 45-47.

[26] M. Breternitz, D. Kowatzki, M. Langenauer, P. Elsner, J.W. Fluhr, Placebocontrolled, double-blind, randomized, prospective study of a glycerol-based emollient on eczematous skin in atopic dermatitis: biophysical and clinical evaluation, Skin Pharmacol. Physiol. 21 (2008) 39-45.

[27] S. Devaux, A. Castela, E. Archier, A. Gallini, P. Joly, L. Misery, S. Aractingi, F. Aubin, H. Bachelez, B. Cribier, D. Jullien, M. Le Maitre, M.A. Richard, J.P. Ortonne, C. Paul, Adherence to topical treatment in psoriasis: a systematic literature review, J. Eur. Acad. Dermatol. Venereol. 26 (Suppl. 3) (2012) 61-67. 